\title{
Applying Grover's algorithm to AES: quantum resource estimates
}

\author{
Markus Grassl $^{1}$, Brandon Langenberg ${ }^{2}$, Martin Roetteler ${ }^{3}$, and Rainer Steinwandt ${ }^{2}$ \\ 1 Universität Erlangen-Nürnberg \& Max Planck Institute for the Science of Light, \\ Günther-Scharowsky-Straße 1, Bau 24, 91058 Erlangen, Germany, Markus . Grass l@ fau . de \\ 2 Florida Atlantic University, 777 Glades Road, Boca Raton, FL 33431, U.S.A., \{blangenb, rsteinwa\}@ fau. edu \\ 3 Microsoft Research, One Microsoft Way, Redmond, WA 98052, U.S.A., martinro@microsoft.com
}

\begin{abstract}
We present quantum circuits to implement an exhaustive key search for the Advanced Encryption Standard (AES) and analyze the quantum resources required to carry out such an attack. We consider the overall circuit size, the number of qubits, and the circuit depth as measures for the cost of the presented quantum algorithms. Throughout, we focus on Clifford $+T$ gates as the underlying fault-tolerant logical quantum gate set. In particular, for all three variants of AES (key size 128, 192, and 256 bit) that are standardized in FIPS-PUB 197, we establish precise bounds for the number of qubits and the number of elementary logical quantum gates that are needed to implement Grover's quantum algorithm to extract the key from a small number of AES plaintext-ciphertext pairs.
\end{abstract}

Keywords: quantum cryptanalysis, quantum circuits, Grover's algorithm, Advanced Encryption Standard

\section{Introduction}

Cryptanalysis is an important area where quantum algorithms have found applications. Shor's seminal work invalidates some well-established computational assumptions in asymmetric cryptography [27], including the hardness of factoring and the computation of discrete logarithms in finite cyclic groups such as the multiplicative group of a finite field. On the other hand, regarding symmetric encryption, the impact of quantum algorithms seems less dramatic. While a quantum version of related key attacks [26] would be a threat for block ciphers provided that quantum access to the encryption function is given, as this requires the ability to generate quantum superpositions of related keys, this attack model is somewhat restrictive. In particular, the related key attack of [26] is not applicable to, say, a context where a small number of plaintext-ciphertext pairs are given and the goal is to identify the encryption key.

It has been known for some time that in principle Grover's search algorithm [15] can be applied to the problem of finding the key: the square root speed-up offered by Grover's algorithm over a classical exhaustive key search seems to be the most relevant quantum cryptanalytic impact for the study of block ciphers. To actually implement such an attack, the Boolean predicate that is queried in Grover's algorithm needs to be realized as a circuit. Perhaps interestingly, even for the most obvious target - the Advanced Encryption Standard [24], which in its 256-bit version has recently been suggested to be quantum-safe [5] - to the best of our knowledge no detailed logical level resource estimate for implementing Grover's algorithm is available. The seemingly simple task of implementing the AES function actually requires some analysis as the circuit implementation is required to be reversible, i.e., it must be possible to implement the operation via an embedding into a permutation. Once a reversible implementation is known, in principle also a quantum implementation can be derived as the set of permutations is a subset of all unitary operations.

Our contribution. We provide reversible circuits that implement the full Advanced Encryption Standard AES- $k$ for each standardized key size (i.e., $k=128,192,256$ ). We establish resource estimates for the number of qubits and the number of Toffoli gates, controlled NOT gates, and NOT gates. See [23] for basic definitions of quantum and reversible logic gates. Furthermore, we consider decompositions of the 
reversible circuits into a universal fault-tolerant gate set that can then be implemented as the set of logical gates. As underlying fault-tolerant gate set we consider the so-called set of Clifford $+T$ gates ${ }^{4}$ This gate set is motivated, e.g., by the fact that this set of gates can be implemented fault-tolerantly on a large set of codes, including the surface code family [14[13] and concatenated CSS codes [28|25]. Clifford gates typically are much cheaper than the $T$-gate which commonly is implemented using state distillation. When breaking down the circuit to the level of $T$-gates we therefore pay attention to reducing the overall $T$-count. See also [3]4] for techniques how to optimize the $T$-count and [2] for techniques that allow to navigate the tradespace between $T$-depth and the number of qubits used. For the particular case of the Toffoli gate we use an implementation that requires $7 T$-gates and several Clifford gates, see [23]3]. There is a probabilistic circuit known that implements the Toffoli gate with only $4 T$-gates [16], however, as the architecture requirements will be stronger in that measurement and feed-forward of classical information is required, we focus on the purely unitary decomposition that requires $7 T$-gates. We remark however, that the only source of $T$ gates in this paper are Toffoli gates, hence it is possible to use Jones' Toffoli factorization mutatis mutandis which leads to all given resource estimates for the $T$-count being multiplied by $4 / 7$ and the requirement of 1 additional ancilla qubit. In our resource estimates we do not to restrict interactions between qubits and leave the implementation, e.g., on a 2D nearest neighbor array for further study, including an investigation of the remaining quantum circuit placement problems [21] that will have to be solved for the logical gate lists that are produced by our approach.

One of our main findings is that the number of logical qubits required to implement a Grover attack on AES is relatively low, namely between around 3,000 and 7,000 logical qubits. However, due to the large circuit depth of unrolling the entire Grover iteration, it seems challenging to implement this algorithm on an actual physical quantum computer, even if the gates are not error corrected. It is worth noting that much of the circuit cost within each Grover iteration originates from the key expansion, i. e., from deriving the round keys and that the overall depth is a direct result of the serial nature of Grover's algorithm.

\section{Preliminaries: Grover's algorithm}

Before going into technicalities of how to implement AES as a quantum circuit, we briefly recall the interface that we need to provide to realize a key search, namely Grover's algorithm [15]. The Grover procedure takes as an input a quantum circuit implementing a Boolean function $f:\{0,1\}^{k} \longrightarrow\{0,1\}$ in the usual way, i.e., via a quantum circuit $U_{f}$ that implements $|x\rangle|y\rangle \mapsto|x\rangle|y \oplus f(x)\rangle$, where $x \in\{0,1\}^{n}$ and $y \in\{0,1\}$. The basic Grover algorithm finds an element $x_{0}$ such that $f\left(x_{0}\right)=1$. Denoting by $H$ the $2 \times 2$ Hadamard transform, the Grover algorithm consists of repeatedly applying the operation $G$ to the initial state $|\psi\rangle \otimes|\varphi\rangle$, where $|\psi\rangle=\frac{1}{\sqrt{2^{k}}} \sum_{x \in\{0,1\}^{k}}|x\rangle,|\varphi\rangle=\frac{1}{\sqrt{2}}(|0\rangle-|1\rangle)$, and where $G$ is defined as

$$
G=U_{f}\left(\left(H^{\otimes k}\left(2|0\rangle\langle 0|-\mathbf{1}_{2^{k}}\right) H^{\otimes k}\right) \otimes \mathbf{1}_{2}\right),
$$

where $|0\rangle$ denotes the all zero basis state of the appropriate size. Overall, $G$ has to be applied a number of $O(\sqrt{N / M})$ times in order to measure an element $x_{0}$ such that $f\left(x_{0}\right)=1$ with constant probability, where $N$ is the total number of candidates, i.e., $N=2^{k}$, and provided that there are precisely $M$ solutions, i.e., $M=|\{x: f(x)=1\}|$; see also [23, Section 6.1.2], [8] for an analysis. If we know that there is only one solution, i.e., $M=1$, this means that we can find a solution by applying $H^{\otimes k+1}$ to the initial state

\footnotetext{
${ }^{4}$ As is common, we do not distinguish between $T=\left(\begin{array}{cc}1 & 0 \\ 0 & \exp (i \pi / 4)\end{array}\right)$ and $T^{\dagger}$-gates.
} 
$|0\rangle^{\otimes k} \otimes|1\rangle$ and then applying $G^{\ell}$, where $\ell=\left\lfloor\frac{\pi}{4} \sqrt{N}\right\rfloor$, followed by a measurement of the entire quantum register which will yield a solution $x_{0}$ with high probability [23, Section 6.1.4], [8].

As we will show in the following section, we can indeed define a function $f$ from the set of possible keys, i.e., $k \in\{128,192,256\}$ for the case of AES, such that there is (plausibly) precisely one solution to the problem of finding the correct key $K$ that was used to encrypt a small set of given plaintext-ciphertext pairs, i.e., we can (plausibly) enforce the situation $M=1$ by defining a suitable function $f$. We remark, however, that it is possible to modify Grover's algorithm in various ways so that it can cope with a larger (but known) number $M>1$ of solutions or even with a completely unknown number of solutions: as mentioned above, if the number $M$ of solutions is known, $O(\sqrt{N / M})$ iterations are enough, however, if the number is unknown, there is an issue that it is not possible to pick the right number of iterations a priori. Nonetheless, there is a variant of the algorithm which finds a solution in expected running time $O(\sqrt{N / M})$ even when the number $M$ of solutions is unknown [8, Section 6].

There are several ways out of this dilemma which we mention briefly for completeness but point out that we did not implement these alternatives: one can first apply a quantum algorithm to count the number of solutions [ [10]8] or one can do an exponential search on the number of iterations [8]9], or one can employ an adaptive schedule in which the Grover operator is changed to an operator that rotates by different angles depending on the index of the iteration [30], thereby driving the oscillation of the quantum state into a bounded region (the "fixed point") which then yields a solution upon measurement.

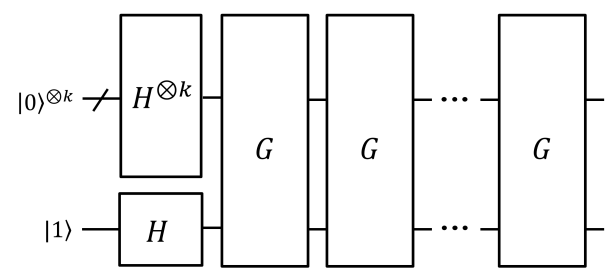

(a)

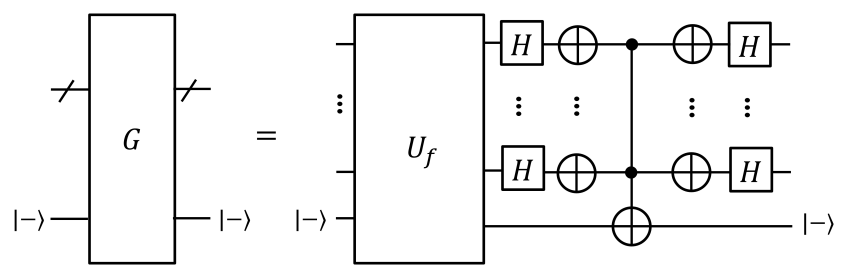

(b)

Fig. 1. (a) Quantum circuit to implement Grover's algorithm. The algorithm consists of creating the equal superposition $\sum_{x}|x\rangle$ in the upper register which for the case of AES has $k=128,192,256$ qubits and a single qubit state $|-\rangle=|0\rangle-|1\rangle$ in the lower register. The operator $G$ is the Grover iterate and is applied a total number of $\left\lfloor\frac{\pi}{4} \sqrt{2^{k}}\right\rfloor$ many times. (b) One round of Grover's algorithm. Shown is the operator $G=U_{f}\left(\left(H^{\otimes k}\left(2|0\rangle\langle 0|-\mathbf{1}_{2^{k}}\right) H^{\otimes k}\right) \otimes \mathbf{1}_{2}\right)$ and its circuit decomposition. Note that the effect of the gates between the two layers of Hadamard gates is to invert the phase of the basis state $|0\rangle$ on the upper $k$ bits (up to a global phase).

Returning to the case of Grover's algorithm with a unique solution, we now study the number of gates and the space requirements needed in order to implement the algorithm. We consider the gates shown in Figure 1 , in particular we first focus on the circuit shown in part (b) of the figure and analyze its complexity. While $H$ is a Clifford operation, besides the operation $U_{f}$ which involves the classical computation of (several) AES functions, we also have to determine the cost $\kappa$ for the operation $(2|0\rangle\langle 0|-1)$ in equation (1). This reduces to the implementation of a $k$-fold controlled NOT gate, where for us $k \in\{128,192,256\}$. The resource estimates for this gates in terms of Toffoli gates can be obtained from [6] to be (as $n \geq 5$ ): $8 k-24$ Toffoli gates which evaluates to $1,000,1,512$, and 2, 024 Toffoli gates per phase operation $\left(2|0\rangle\langle 0|-\mathbf{1}_{2^{k}}\right)$, respectively. For the number of Clifford $+T$ gates (counting only $T$ s) one could directly apply an upper bound by multiplying $\kappa$ with 7, however, one can derive a slightly better bound: as shown in [29] (see also [20]), one can employ phase cancellations and show an upper bound of $32 k-84$ for a $k$-fold controlled 
NOT gate, i.e., we obtain 4,012, 6,060, and 8, 108 for the $T$-count per phase operation for the three key sizes $k \in\{128,192,256\}$.

We spend the rest of the paper to obtain estimates for $f:\{0,1\}^{k} \rightarrow\{0,1\}$ which proceeds by first mapping $K \mapsto\left(\operatorname{AES}_{K}\left(m_{1}\right), \ldots, \operatorname{AES}_{K}\left(m_{r}\right)\right)$ and then computing the equality function of the resulting vector with the given ciphertexts $c_{1}, \ldots, c_{r}$, where $c_{i} \in\{0,1\}^{128}$. In other words, we define the value of $f$ on a given input key $K \in\{0,1\}^{k}$ (where $k \in\{128,192,256\}$ ) as follows:

$$
f(K):=\left(\operatorname{AES}_{K}\left(m_{1}\right)=c_{1}\right) \wedge \ldots \wedge\left(\operatorname{AES}_{K}\left(m_{r}\right)=c_{r}\right) .
$$

As argued below, it is plausible that $r=3,4,5$ are sufficient for the three standardized AES key sizes. The equality function can be implemented by a multiply controlled NOT gate that has $128 \mathrm{r}$ (many controls where $r=3,4,5$ ) and a single target. Using the above formulas this leads to Toffoli counts of 3, 048, 4, 072, and 5,096, respectively, as well as $T$-counts of $12,204,16,300$, and 20,396, respectively. We return to the question of providing exact quantum resource estimates for Grover's algorithm in Section 3.4 after the implementation details of the "oracle" function $U_{f}$ have been derived in the subsequent sections.

\section{Implementing the Boolean predicate-testing a key}

An essential component needed in Grover's algorithm is a circuit which on input a candidate key $|K\rangle$ indicates if this key is equal to the secret target key or not. To do so, the idea is to simply encrypt some (fixed) plaintext under the candidate key and compare the result with the (assumed to be known) corresponding ciphertext under the secret target key.

\subsection{Ensuring uniqueness of the solution}

As AES always operates on 128-bit plaintexts, at least for 192-bit and 256-bit keys we have to assume that fixing a single plaintext-ciphertext pair is not sufficient to determine a secret key uniquely.

Arguing with the strict avalanche criterion [19|11] exactly in the same way as in [26, Section 2.1], we can plausibly assume that for every pair of keys $\left(K, K^{\prime}\right) \in\{0,1\}^{k \times k}$ with $K \neq K^{\prime}$ the condition

$$
\left(\operatorname{AES}_{K}\left(m_{1}\right), \ldots, \operatorname{AES}_{K}\left(m_{r}\right)\right) \neq\left(\operatorname{AES}_{K^{\prime}}\left(m_{1}\right), \ldots, \operatorname{AES}_{K^{\prime}}\left(m_{r}\right)\right)
$$

holds for some suitable collection of plaintexts $m_{1}, \ldots, m_{r}$. The reason for this is that, for a fixed plaintext, when flipping a bit in the secret key, then each bit of the corresponding ciphertext should change with probability $1 / 2$. Hence, for $r$ simultaneous plaintext-ciphertext pairs that are encrypted under two secret keys $K^{\prime} \neq K$ we expect to get different results with probability about $1-2^{-r n}$, if the plaintexts are pairwise different, where $n$ denotes the length of the message. Hence out of a total of $2^{2 k}-2^{k}$ key pairs $\left(K, K^{\prime}\right)$ with $K \neq K^{\prime}$, about $\left(2^{2 k}-2^{k}\right) \cdot 2^{-r n} \leq 2^{2 k-r n}$ keys $K^{\prime} \neq K$ are expected to give the same encryptions. Hence it seems plausible to estimate that

$$
r>\lceil 2 k / n\rceil
$$

plaintexts suffice to ensure that for every $K^{\prime} \neq K$ at least one separating plaintext is available. As AES has 128-bit plaintexts we have that $n=128$, i.e., Eq. (2) implies that for key length $k$ the adversary has $r>\lceil 2 k / 128\rceil$ plaintext-ciphertexts pairs $\left(m_{1}, r_{1}\right), \ldots,\left(m_{r}, c_{r}\right)$ for the target key available. In other words, to characterize the secret target key uniquely, we assume that $r=3$ (AES-128), $r=4$ (AES-192), and $r=5$ (AES-256) suitable plaintext-ciphertext pairs are known by the adversary. 


\subsection{Reversible and quantum circuits to implement AES}

We assume that the reader is familiar with the basic components of AES. For a detailed specification of AES we refer to FIPS-PUB 197 [24]. To realize this round-oriented block cipher as a reversible circuit over the Toffoli gate set, respectively as a quantum circuit over the Clifford $+T$ gate set, we need to take care of the key expansion, which provides all needed 128-bit round keys, as well as the individual rounds. While the number of rounds depends on the specific key length $k$, the four main functions-AddRoundKey, MixColumns, ShiftRows, and SubBytes-that are used to modify the 128-bit internal state of AES are independent of $k$.

First, we discuss the realization of these four functions, before going into details of combining them with the key expansion into complete round functions and a full AES. In our design choices, we tried to keep the number of qubits low, even when this results in a somewhat larger gate complexity. For instance, to implement the $\mathbb{F}_{256}$-multiplications within SubBytes, we opted for a multiplier architecture requiring less qubits, but more Clifford and more $T$-gates.

3.2.1 Circuits for the basic AES operations The internal AES state consists of 128 bits, organized into a rectangular array of $4 \times 4$ bytes. We will devote 128 qubits to hold the current internal state.

AddRoundKey. In the implementation of the key expansion, we ensure that the current round key is available on 128 dedicated wires. Implementing the bit-wise XOR of the round key then reduces to 128 CNOT gates which can all be executed in parallel.

MixColumns. Since MixColumns operates on an entire column of the state or 32 (qu)bits at a time, the matrix specified in [24] was used to generate a $32 \times 32$ matrix. An LUP-type decomposition was used on this $32 \times 32$ matrix in order to compute this operation in place with 277 CNOT gates and a total depth of 39. Example 1 offers a similar but smaller version of an LUP-type decomposition as we used.

ShiftRows. As ShiftRows amount to a particular permutation of the current AES state, we do not have to add any gates to implement this operation as it corresponds to a permutation of the qubits. Instead, we simply adjust the position of subsequent gates to make sure that the correct input wire is used.

SubBytes. This operation replaces one byte of the current state with a new value. For a classical implementation, a look-up table can be an attractive implementation option, but for our purposes, explicitly calculating the result of this operation seems the more resource friendly option. Treating a state byte as element $\alpha \in \mathbb{F}_{2}[x] /\left(1+x+x^{3}+x^{4}+x^{8}\right)$, first the multiplicative inverse of $\alpha$ (leaving 0 invariant) needs to be found. This is followed by an affine transformation. To find $\alpha^{-1}$ we adopt the idea of [1] to build on a classical Itoh-Tsujii multiplier, but we work with in-place matrix multiplications. Specifically, we compute

$$
\alpha^{-1}=\alpha^{254}=\left(\left(\alpha \cdot \alpha^{2}\right) \cdot\left(\alpha \cdot \alpha^{2}\right)^{4} \cdot\left(\alpha \cdot \alpha^{2}\right)^{16} \cdot \alpha^{64}\right)^{2},
$$

exploiting that all occurring exponentiations are $\mathbb{F}_{2}$-linear. Using again an LUP-type decomposition, the corresponding matrix-multiplication can be realized in-place, using CNOT gates only. And by adjusting the positions of subsequent gates accordingly, realizing the permutation is for free, no gates need to be introduced for this.

Example 1. Squaring in $\mathbb{F}_{2}[x] /\left(1+x+x^{3}+x^{4}+x^{8}\right)$ can be expressed as multiplying the coefficient vector from the left with 


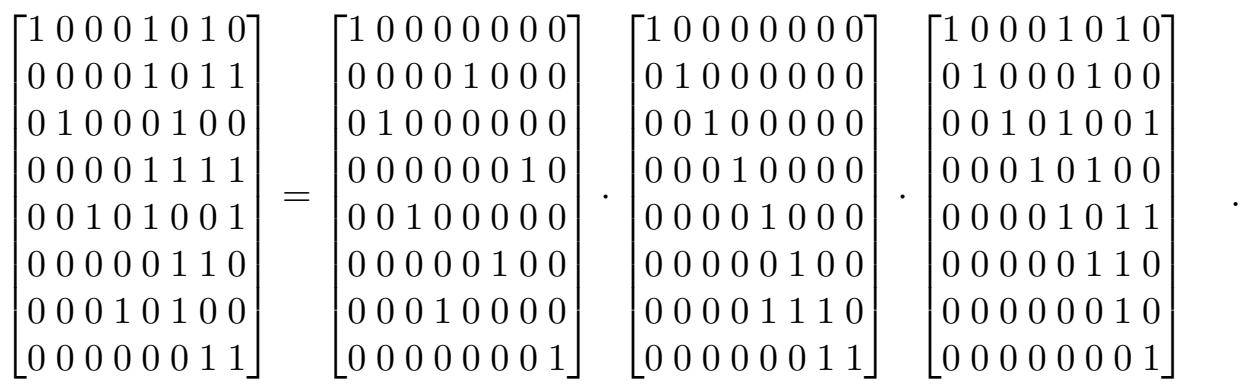

From this, we see that in-place-squaring can be implemented with only twelve CNOT gates. The resulting circuit is shown in Figure 2 .

To realize the six multiplications in Equation (3), we use a general purpose multiplier in the underlying binary field. We opted for a design by Maslov et al. [22], which requires less than $60 \%$ of the number of qubits than a more recent design in [18]. This comes at the cost of an increased gate complexity, however, and a different design choice could be considered. For the specific polynomial basis representation of $\mathbb{F}_{256}$ at hand, Maslov et al.'s design, requires 64 Toffoli plus 21 CNOT gates, which with Amy et al. [3] translates into $64 \cdot 7=448 T$ - plus $64 \cdot 8+21=533$ Clifford gates.

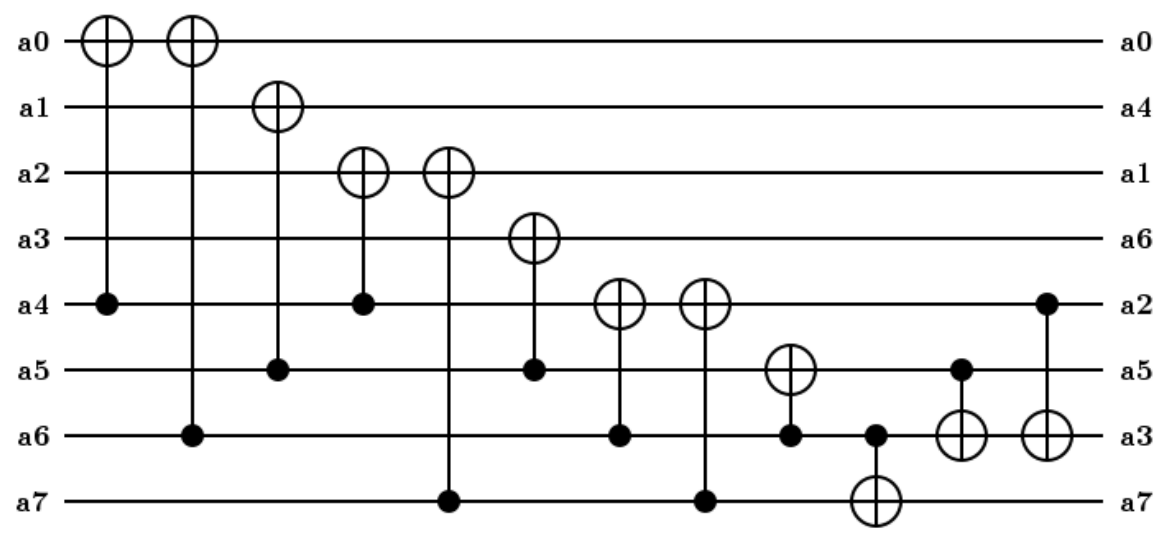

Fig. 2. Squaring in $\mathbb{F}_{2}[x] /\left(1+x+x^{3}+x^{4}+x^{8}\right)$

Noticing that three of the multiplications in Equation (3) are actually duplicates, it turns out that four multiplications suffice in order to implement the inversion. Trying to reduce the number of total qubits required at each step, the actual calculation of computing $\alpha^{-1}$ fits into 40 qubits total, producing $|\alpha\rangle,|\alpha\rangle^{-1}$, and twenty-four reinitialized qubits as output. To do so, and reinitialize qubits, we invest twelve linear transformations and eight $\mathbb{F}_{256}$-multiplications, totalling $3584 T$-gates and 4539 Clifford gates.

Once $\alpha^{-1}$ is found, the affine transformation specified in [24] must be computed, which can be done with an LUP-type decomposition; four uncontrolled NOT gates take care of the vector addition after multiplication with a matrix. In total one 8-bit S-box requires $3584 T$-gates and 4569 Clifford gates. 
SubBytes-an alternative implementation minimizing qubits. The inversion $\alpha \mapsto \alpha^{-1}$ (where 0 is mapped to 0) can be seen as a permutation on $\mathbb{F}_{256}$. This permutation is odd, while quantum circuits with NOT, CNOT, and Toffoli gates on $n>3$ qubits generate the full alternating group $A_{2^{n}}$ of even permutations. Hence we have to use one ancilla qubit, i.e., nine qubits in total. The task is then to express a permutation on 512 points in terms of the generators corresponding to the NOT, CNOT, and Toffoli gates. While computer algebra systems like Magma [7] have built-in functions for this, the resulting expressions will be huge. In order to find a short factorization, we compute a stabilizer chain and corresponding transversals using techniques similar to those described in [12]. We use a randomized search to find short elements in each transversal. As it is only relevant to implement the exact function when the ancilla qubit is in the state $|0\rangle$, we choose the first 256 points in the basis for the permutation group as those with the ancilla in the state $|0\rangle$, and the remaining 256 points as those with the ancilla in the state $|1\rangle$. This allows to compute a factorization modulo permutations of the last 256 points. With this approach, we found a circuit with no more than 9695 $T$-gates and 12631 Clifford gates, less than three times more gates than the version above, but using only 9 instead of 40 qubits in total.

3.2.2 Key Expansion Standard implementation of the key expansion for AES- $k(k=128,192,256)$ separates the original $k$-bit key into 4, 6 or 8 words of length 32, respectively and must expand the $k$-bit key into forty-four words for $k=128$, fifty-two words for $k=192$ and sixty words for $k=256$. Each AES key expansion uses the same operations and there are only slight differences in the actual round key construction. The operations are RotWord, a simple rotation, SubBytes, and Rcon[i], which adds $x^{i-1} \in \mathbb{F}_{256}$ to the first byte of each word.

While the three different versions of AES employ up to 14 rounds of computation, the key expansion is independent of the input. The words created by the key expansion were divided into two categories: the words needing SubBytes in their computation and those that do not. The words not involving SubBytes can be recursively constructed from those that do by a combination of XORings making them simple to compute as needed, saving up to $75 \%$ of the storage cost of the key expansion. The most expensive of these is word 41 or $w_{41}$ in AES-128 which is constructed by XORing 11 previous words costing 352 CNOT gates and a total depth of 11 .

\begin{tabular}{cccccccc}
\hline \hline & \multicolumn{3}{c}{ \#gates } & \multicolumn{2}{c}{ depth } & \multicolumn{2}{c}{ \#qubits } \\
& NOT & CNOT & Toffoli & $T$ & overall & storage & ancillae \\
\hline 128 & 176 & 21,448 & 20,480 & 5,760 & 12,636 & 320 & 96 \\
192 & 136 & 17,568 & 16,384 & 4,608 & 10,107 & 256 & 96 \\
256 & 215 & 27,492 & 26,624 & 7,488 & 16,408 & 416 & 96 \\
\hline \hline
\end{tabular}

Table 1. Quantum resource estimates for the key expansion phase of AES- $k$, where $k \in\{128,192,256\}$.

Since SubBytes is costly, the remaining words are stored as they are constructed. In a classical AES implementation, these words (every fourth or sixth) are produced by starting with the previous word, however in this construction the previous word must be constructed, and removed, as needed. For example, in AES-128, to construct $w_{8}$, first $w_{7}$ must be constructed as follows: $w_{7}=w_{4} \oplus w_{3} \oplus w_{2} \oplus w_{1}$.

This can be done on the previously constructed word (here $w_{4}$ ) saving qubits, gates, and depth. Since the construction of $w_{8}$ involves the use of $w_{4}$ the above process needs to be repeated to be removed before the end of construction of $w_{8}$. For the construction of these words, similar to ShiftRows, RotWord can be 
eliminated if the position of the gates is shifted to use the correct wires. Since SubWord applies SubBytes to each byte of the word independently, each of the four SubBytes computations can be done concurrently.

Example 2. Below is the construction of $w_{8}$. Notice that $w_{7}$ is constructed on top of $w_{4}$.

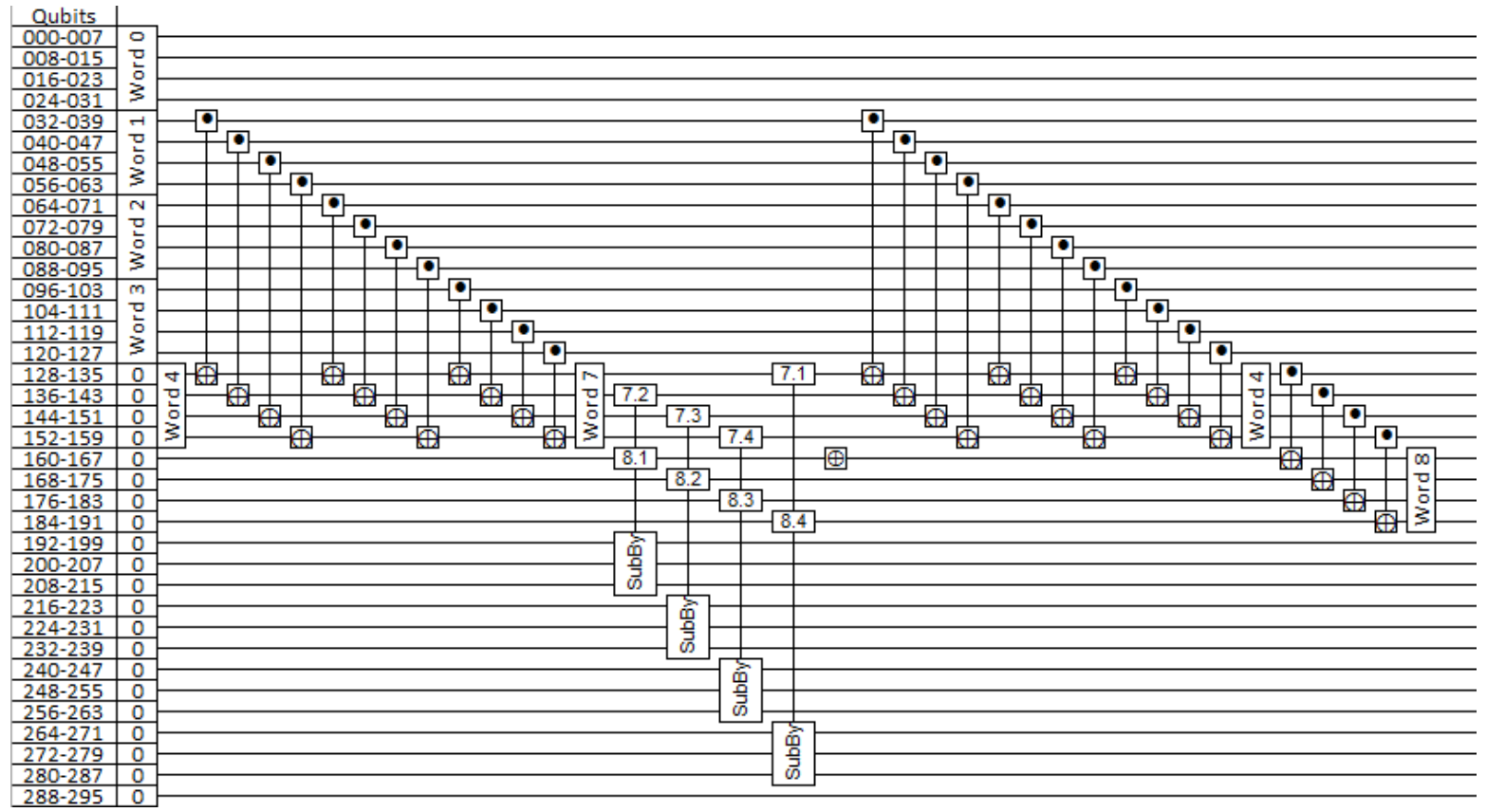

To allow each of the four SubBytes routines per round to perform simultaneously, 96 auxilary qubits would be needed, along with the 32 needed to store the new word. With each word constructed requiring the previous word be constructed first, we did not reduce the depth further. Computation costs are listed in Table1 (the listed qubit costs do not include storing the original key).

3.2.3 AES Rounds. AES starts with a simple whitening step-XORing the input with the first four words of the key. Since, in this case, the input is a fixed value, and adding a fixed value can be done by simply flipping bits, approximately 64 uncontrolled NOT gates are used on the first four key words to start round one. This can be reversed later when needed, but saves 128 qubits. If this is not the case, then 128 qubits are needed to store the input and 128 CNOT gates can be used to compute this step. While the 10, 12, or 14 rounds of AES all apply the same basic functions, the circuit structure differs slightly per round to reduce qubits and depth. SubBytes must be computed 16 times per round, requiring 384 auxiliary qubits for all to be done simultaneously or an increase in depth is needed. Using only the minimum 24 auxiliary qubits and the 128 qubits needed to store the result, it was noticed that all 16 SubBytes calculations per round could be done with a maximum depth of 8 SubBytes cycles.

Since SubBytes is not done in place, and AES- $k$ requires 128 qubits per round, the computation takes 128 qubits times the number of rounds per AES, in addition to the number of qubits needed to store the original key. This number can be reduced by reversing steps between computations to clear qubits for future use. Once SubBytes has been applied, the input can be removed by reversing enough steps (but the output could not be removed as its counterpart (inverse) is gone). Since AES-128 employs 10 rounds, using 512 qubits for storage and 24 auxiliary qubits, allows the reverse process to be applied three times. For AES-192 and AES-256, we used 640 qubits for storage since we did not manage to have three rounds of reversing on 536 qubits. 
Example 3. The reverse process representation for AES-128. Notice this method leaves Round 4, Round 7 and Round 9 with no way to be removed unless the entire process is reversed.

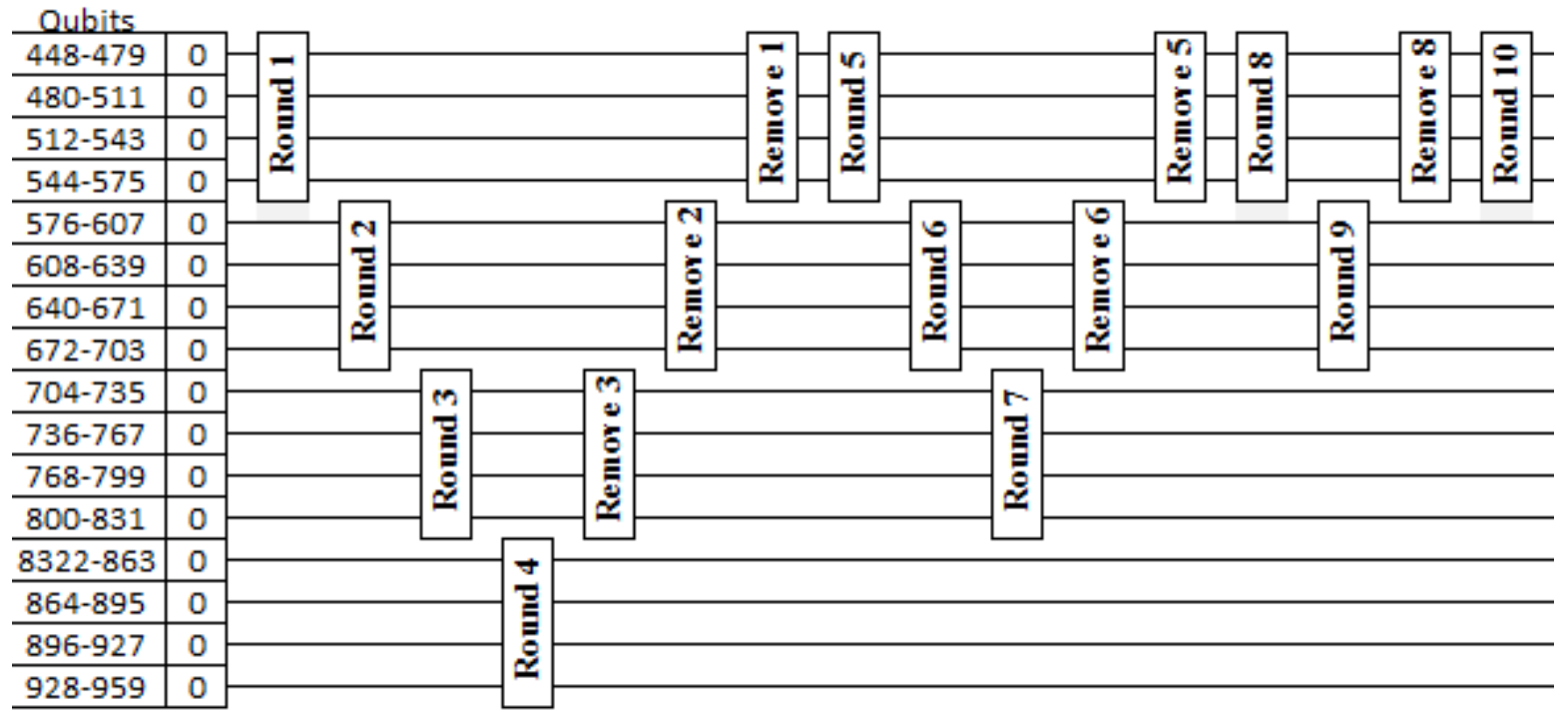

For AES-192 and AES-256 the reversing process is done after rounds five, nine and twelve, requiring only 128 qubits more than AES-128.

As stated, ShiftRows is for free and using an LUP-type decomposition for MixedColumns allows this process to be done in place using 277 CNOT gates with a maximum depth of 39. To compute all 10 rounds of AES-128, 536 qubits were needed, 664 qubits were used to compute the 12 rounds of AES-192 and 14 rounds of AES-256.

The XORing of the round keys can be done directly on top of the input for each round. If the round key needed is already constructed, 128 CNOT gates with a depth of 1 are used to complete the round. If the round key is not already constructed and thus a combination of constructed keys, then it only requires this process to be done multiple times. AES-128 requires this to be done 11 times (the most) in the case of $w_{41}$, increasing the depth and CNOT gate count by at most 11.

\subsection{Resource estimates: reversible AES implementation}

The numbers listed in the three tables below show the costs in gates, depth and qubits to achieve the output of each AES- $k$ system.

\begin{tabular}{cccccc}
\hline \hline & \multicolumn{2}{c}{ \#gates } & \multicolumn{2}{c}{ depth } & \#qubits \\
& $T$ & Clifford & $T$ & overall & \\
\hline Initial & 0 & 0 & 0 & 0 & 128 \\
Key Gen & 143,360 & 185,464 & 5,760 & 12,626 & 320 \\
10 Rounds & 917,504 & $1,194,956$ & 44,928 & 98,173 & 536 \\
\hline Total & $1,060,864$ & $1,380,420$ & 50,688 & 110,799 & 984 \\
\hline \hline
\end{tabular}

Table 2. Quantum resource estimates for the implementation of AES-128. 


\begin{tabular}{cccccc}
\hline \hline & \multicolumn{2}{c}{ \#gates } & \multicolumn{2}{c}{ depth } & \#qubits \\
& $T$ & Clifford & $T$ & overall & \\
\hline Initial & 0 & 0 & 0 & 0 & 192 \\
Key Gen & 114,688 & 148,776 & 4,608 & 10,107 & 256 \\
12 Rounds & $1,089,536$ & $1,418,520$ & 39,744 & 86,849 & 664 \\
\hline Total & $1,204,224$ & $1,567,296$ & 44,352 & 96,956 & 1,112 \\
\hline \hline
\end{tabular}

Table 3. Quantum resource estimates for the implementation of AES-192. The lower gate count in Key Gen and the lower depth, when compared to AES-128, arises from using the additional available space to store intermediate results and to parallelize parts of the circuit.

\begin{tabular}{cccccc}
\hline \hline & \multicolumn{2}{c}{ \#gates } & \multicolumn{2}{c}{ depth } & \#qubits \\
& $T$ & Clifford & $T$ & overall & \\
\hline Initial & 0 & 0 & 0 & 0 & 256 \\
Key Gen & 186,368 & 240,699 & 7,488 & 16,408 & 416 \\
14 Rounds & $1,318,912$ & $1,715,400$ & 52,416 & 114,521 & 664 \\
\hline Total & $1,505,280$ & $1,956,099$ & 59,904 & 130,929 & 1,336 \\
\hline \hline
\end{tabular}

Table 4. Quantum resource estimates for the implementation of AES-256.

\subsection{Resource estimates: Grover algorithm}

From the discussion in the previous sections we obtain a reversible circuit for computing $\operatorname{AES}_{K}\left(m_{i}\right)$, i.e., a circuit $\mathcal{C}$ that implements the operation $|K\rangle|0\rangle \mapsto|K\rangle\left|\operatorname{AES}_{K}\left(m_{i}\right)\right\rangle$. The overall circuit to implement $U_{f}$ is shown in Figure 3. The AES layer can be applied in parallel, however, as the used ancilla qubits have to be returned clean after each round, we have to uncompute each AES box within each round. Hence the depth (and $T$-depth) increases by a factor of 2 within each invocation of $U_{f}$. The total number of gates (and $T$-gates) on the other hand increases by a factor of $2 r$ as all boxes have now to be counted. The number of qubits is given by $r$ times the number of qubits within each AES box.

Once the AES boxes have been computed, the result is compared with the given ciphertexts $c_{1}, \ldots, c_{r}$. Note that as AES operates on plaintexts/ciphertexts of length 128 we have that $c_{i} \in\{0,1\}^{128}$ throughout. The comparison is done by a multiply controlled NOT gate and the controls are either 0 or 1 depending on the bits of $c_{i}$. This is denoted by the superscript $c_{i}$ on top of the controls in Figure 3. We can now put everything together to estimate the cost for Grover's algorithm based on the AES- $k$ resource estimates given in the previous section: denoting by $s_{k}$ the total number of qubits, $t_{k}$ the total number of $T$-gates, $c_{k}$ the total number of Clifford gates, $\delta_{k}$ the overall $T$-depth and $\Delta_{k}$ the overall depth, where $k=128,192,256$, then we obtain the following estimates for the overall Grover algorithm. The space requirements are $3 s_{128}+1$ qubits for AES-128, $4 s_{192}+1$ qubits for AES-192, and $5 s_{256}+1$ qubits for AES-256.

Regarding the time complexity, we obtain that per Grover iteration we need $6 t_{128}$ many $T$-gates for AES-128 plus the number of $T$-gates needed for the 384 -fold controlled NOT inside $U_{f}$ and the 128 -fold controlled NOT to implement the phase $(2|0\rangle\langle 0|-1)$. We estimated the $T$-counts of these two operations earlier to be 12,204 and 1,000 respectively. Overall, we have to perform $\left\lfloor\frac{\pi}{4} 2^{k / 2}\right\rfloor$ iterations, i.e., we obtain 


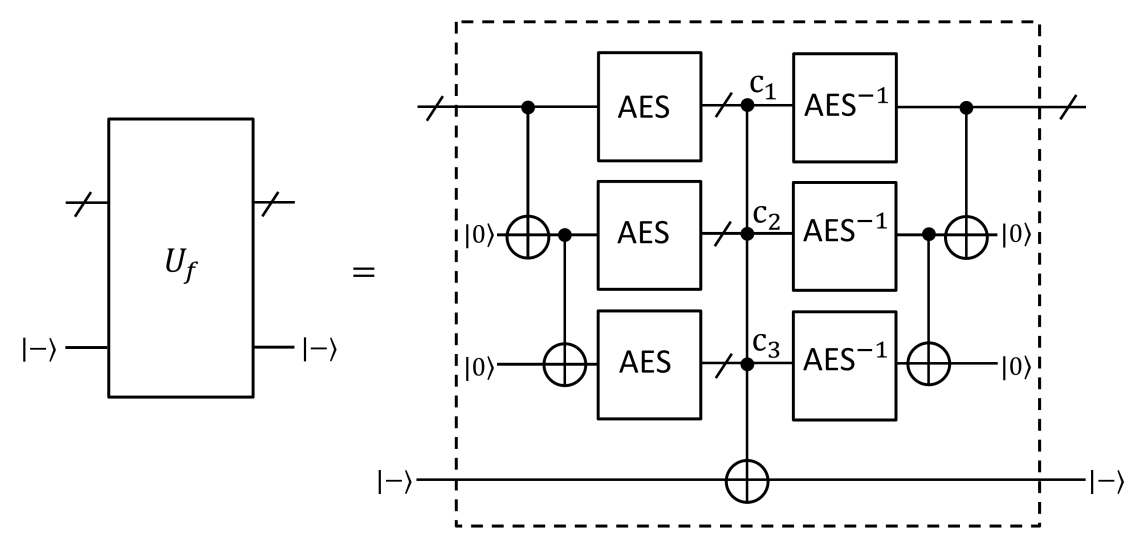

Fig. 3. The reversible implementation of the function $U_{f}$ is shown in further detail. In this case the key size $k=128$ is considered for which $r=3$ invocations of AES suffice in order to make the target key unique. For the cases of $k=192$ the number of parallel AES boxes increases to $r=4$ and for $k=256$ to $r=5$, however, the overall structure of the circuit is common to all key sizes.

for the overall $T$-gate count for Grover on AES-128 the estimate of

$$
\left\lfloor\frac{\pi}{4} 2^{64}\right\rfloor \cdot\left(6 t_{128}+13,204\right)=9.24 \cdot 10^{25}=1.19 \cdot 2^{86}
$$

many $T$-gates. Similarly, we can estimate the number of Clifford gates which for simplicity we just assume to be $6 c_{128}$, ignoring some of the Clifford gates used during the rounds. For AES-192 we have to perform $\left\lfloor\frac{\pi}{4} 2^{96}\right\rfloor$ iterations and for AES-256 we have to perform $\left\lfloor\frac{\pi}{4} 2^{128}\right\rfloor$ iterations. For the $T$-count of the controlled operations we obtained $16,300+1,512=17,812$ and $20,396+2024=22,420$ earlier. Overall, this gives for Grover on AES-192 the estimate of $3.75 \cdot 10^{36}=1.81 \cdot 2^{114}$ many $T$-gates and for Grover on AES-256 the estimate of $4.03 \cdot 10^{45}=1.41 \cdot 2^{151}$ many $T$-gates. For the overall circuit depth we obtain the number of rounds times 2 times $\delta_{k}$, respectively $\Delta_{k}$, ignoring some of the gates which do not contribute significantly to the bottom line. The overall quantum resource estimates are given in Table 5.

\begin{tabular}{cccccc}
\hline \hline & \multicolumn{2}{c}{ \#gates } & \multicolumn{2}{c}{ depth } & \#qubits \\
$k$ & $T$ & Clifford & $T$ & overall & \\
\hline 128 & $1.19 \cdot 2^{86}$ & $1.55 \cdot 2^{86}$ & $1.06 \cdot 2^{80}$ & $1.16 \cdot 2^{81}$ & 2,953 \\
192 & $1.81 \cdot 2^{118}$ & $1.17 \cdot 2^{119}$ & $1.21 \cdot 2^{112}$ & $1.33 \cdot 2^{113}$ & 4,449 \\
256 & $1.41 \cdot 2^{151}$ & $1.83 \cdot 2^{151}$ & $1.44 \cdot 2^{144}$ & $1.57 \cdot 2^{145}$ & 6,681 \\
\hline \hline
\end{tabular}

Table 5. Quantum resource estimates for Grover's algorithm to attack AES- $k$, where $k \in\{128,192,256\}$.

\section{Conclusion}

When realizing AES, only SubBytes involves $T$-gates. Moreover, SubBytes is called a minimum of 296 times as in AES-128 and up to 420 times in AES-256. As shown above, for all three standardized key lengths, this results in quantum circuits of quite moderate complexity. So it seems prudent to move away from 128-bit keys when expecting the availability of at least a moderate size quantum computer. 
As mentioned in the context of the discussion about Grover's algorithm in the presence of an unknown number of solutions, the implementation of the algorithms in [10] for quantum counting, [9] for general amplitude amplifications, and [30] for fixed-point quantum search might lead to space-time tradeoff implementations of the function $f$. This might in particular be beneficial for the circuit mentioned in [30] as this does not incur a space overhead and can deal with an unknown number of solutions, provided an upper bound on the number of solutions is known a priori. We leave the question of providing quantum resource estimations for attacking AES and other block ciphers by means of such fixed-point versions of Grover's algorithm for future work. Also an interesting area of future research is the resource cost estimation of recently proposed quantum linear and differential cryptanalysis [17].

\section{Acknowledgments}

BL and RS were supported by AFRL/RIKF Award No. FA8750-15-2-0047. RS was also supported by NATO's Public Diplomacy Division in the framework of "Science for Peace," Project MD.SFPP 984520. The authors thank Schloss Dagstuhl for hosting Seminar 15371, during which part of this work was done.

\section{References}

1. Brittanney Amento, Martin Rötteler, and Rainer Steinwandt. Efficient quantum circuits for binary elliptic curve arithmetic: reducing T-gate complexity. Quantum Information \& Computation, 13:631-644, 2013.

2. Matthew Amy, Dmitri Maslov, and Michele Mosca. Polynomial-time $T$-depth optimization of Clifford $+T$ circuits via matroid partitioning. IEEE Transactions on Computer-Aided Design of Integrated Circuits and Systems, 33(10):1476-1489, 2014. arXiv:1303.2042

3. Matthew Amy, Dmitri Maslov, Michele Mosca, and Martin Roetteler. A meet-in-the-middle algorithm for fast synthesis of depth-optimal quantum circuits. IEEE Transactions on Computer-Aided Design of Integrated Circuits and Systems, 32(6):818830, June 2013. For a preprint version see [4].

4. Matthew Amy, Dmitri Maslov, Michele Mosca, and Martin Roetteler. A meet-in-the-middle algorithm for fast synthesis of depth-optimal quantum circuits. arXiv:quant-ph/1206.0758v3, January 2013. Available at http://arxiv.org/abs/ $1206.0758 \mathrm{v} 3$

5. Daniel Augot, Lejla Batina, Daniel J. Bernstein, Joppe Bos, Johannes Buchmann, Wouter Castryck, Orr Dunkelmann, Tim Güneysu, Shay Gueron, Andreas Hülsing, Tanja Lange, Mohamed Saied Emam Mohamed, Christian Rechberger, Peter Schwabe, Nicolas Sendrier, Frederik Vercauteren, and Bo-Yin Yang. Initial recommendations of long-term secure postquantum systems. Available at pqcrypto.eu.org/docs/initial-recommendations.pdf 2015.

6. Adriano Barenco, Charles H. Bennett, Richard Cleve, David P. DiVincenzo, Norman Margolus, Peter W. Shor, Tycho Sleator, John Smolin, and Harald Weinfurter. Elementary gates for quantum computation. Physical Review A, 52(5):3457-3467, 1995.

7. Wieb Bosma, John Cannon, and Catherine Playoust. The Magma algebra system. I. The user language. Journal of Symbolic Computation, 24:235-265, 1997.

8. Michel Boyer, Gilles Brassard, Peter Høyer, and Alain Tapp. Tight bounds on quantum searching. Fortschritte der Physik, 46:493-506, 1998. arXiv:quant-ph/9605034

9. Gilles Brassard, Peter Høyer, Michele Mosca, and Alain Tapp. Quantum amplitude amplification and estimation. AMS Contemporary Mathematics, 305:53-74, 2002. arXiv:quant-ph/0005055

10. Gilles Brassard, Peter Høyer, and Alain Tapp. Quantum counting. In Automata, Languages and Programming, 25th International Colloquium (ICALP'98), pages 820-831, 1998.

11. Edward Dawson, Helen Gustafson, and Anthony N. Pettitt. Strict key avalanche criterion. Australasian Journal of Combinatorics, 6:147-153, 1992.

12. Egner, Sebastian and Püschel, Markus. Solving puzzles related to permutations groups. In Proc. International Symposium on Symbolic and Algebraic Computation (ISSAC'98), pages 186-193, 1998.

13. Austin G. Fowler, Matteo Mariantoni, John M. Martinis, and Andrew N. Cleland. Surface codes: Towards practical large-scale quantum computation. Phys. Rev. A, 86:032324, 2012. arXiv:1208.0928

14. Austin G. Fowler, Ashley M. Stephens, and Peter Groszkowski. High threshold universal quantum computation on the surface code. Physical Review A, 80:052312, 2009. 
15. Lov K. Grover. A fast quantum mechanical algorithm for database search. In Gary L. Miller, editor, Proceedings of the Twenty-Eighth Annual ACM Symposium on the Theory of Computing (STOC 1996), pages 212-219. ACM, 1996.

16. N. Cody Jones. Novel constructions for the fault-tolerant toffoli gate. Physical Review A, 87:022328, 2013.

17. Marc Kaplan, Gaëtan Leurent, Anthony Leverrier, and María Naya-Plasencia. Quantum differential and linear cryptanalysis. arXiv: 1510.05836

18. Shane Kepley and Rainer Steinwandt. Quantum circuits for $\mathbb{F}_{2^{n}}$-multiplication with subquadratic gate count. Quantum Information Processing, 14(7):2373-2386, 2015.

19. Alan G. Konheim. Cryptography. A Primer. John Wiley and Sons, 1981.

20. Dmitri Maslov. On the advantages of using relative phase Toffolis with an application to multiple control Toffoli optimization. arXiv: 1508.03273

21. Dmitri Maslov, Sean M. Falconer, and Michele Mosca. Quantum circuit placement: optimizing qubit-to-qubit interactions through mapping quantum circuits into a physical experiment. In Proceedings of the 44th Design Automation Conference DAC 2007, pages 962-965. ACM, 2007.

22. Dmitri Maslov, Jimson Mathew, Donny Cheung, and Dhiraj K. Pradhan. On the design and optimization of a quantum polynomial-time attack on elliptic curve cryptography. arXiv:0710.1093 2, February 2009. Available at http: //arxiv.org/abs/0710.1093v2.

23. Michael A. Nielsen and Issac L. Chuang. Quantum Computation and Quantum Information. Cambridge University Press, Cambridge, UK, 2000.

24. NIST. Specification for the ADVANCED ENCRYPTION STANDARD (AES). Federal Information Processing Standards Publication 197, November 2001.

25. Ben W. Reichardt. Quantum universality by state distillation. Quantum Information and Computation, 9:1030-1052, 2009.

26. Martin Roetteler and Rainer Steinwandt. A note on quantum related-key attacks. Information Processing Letters, 115(1):4044, 2015.

27. Peter W. Shor. Polynomial-time algorithms for prime factorization and discrete logarithms on a quantum computer. SIAM Journal on Computing, 26(5):1484-1509, 1997.

28. Andrew M. Steane. Overhead and noise threshold of fault-tolerant quantum error correction. Phys. Rev. A, 68:042322, 2003. quant-ph/0207119.

29. Nathan Wiebe and Martin Roetteler. Quantum arithmetic and numerical analysis using Repeat-Until-Success circuits. arXiv:1406.2040

30. Theodore J. Yoder, Guang Hao Low, and Isaac L. Chuang. Fixed-point quantum search with an optimal number of queries. Physical Review Letters, 113:210501, 2014. 